\title{
Pressure Behaviour of Single Wall Carbon Nanotube Bundles and Fullerenes: A Raman Study
}

\author{
A.K. Sood (a, b), Pallavi V. Teresdesai (a), D.V.S. Muthu (a), Rahul Sen (b), \\ A. Govindaraj (b), and C.N.R. RaO (b) \\ (a) Department of Physics, Indian Institute of Science, Bangalore-560 012, India \\ (b) Jawaharlal Nehru Center for Advanced Scientific Research, Jakkur Campus, \\ Jakkur P.O., Bangalore-560 064, India
}

\begin{abstract}
We shall discuss our recent high pressure Raman studies on single wall carbon nanotube (SWNT) bundles and compare them with pressure-induced amorphous and polymeric phases of crystalline $\mathrm{C}_{60}$ and $\mathrm{C}_{70}$. Our high pressure Raman studies on SWNT bundles carried out upto $25.9 \mathrm{GPa}$ show that the intensities of both the radial modes $\left(\omega \approx 170 \mathrm{~cm}^{-1}\right)$ as well as tangential modes (around $1590 \mathrm{~cm}^{-1}$ ) decrease significantly with pressure, so much that the radial modes cannot be observed beyond 2.6 GPa. Most interestingly, the frequency of the dominant tangential mode increases upto $11 \mathrm{GPa}$, then decreases till $16 \mathrm{GPa}$ and again increases. Raman spectra were resolved into four modes upto $10 \mathrm{GPa}$ beyond which only one or two modes could be fitted to the recorded spectra. The pressure-softening of the mode between 11 and $16 \mathrm{GPa}$ as well as other features of the Raman spectra are reversible in the decreasing pressure cycle. These results, though not understood at present, can be associated with the distortion of the circular cross-section of the tubes in the bundle, eventually leading to a possible transition of the SWNT bundle to graphite like carbon at $11 \mathrm{GPa}$ which is completed at $16 \mathrm{GPa}$. This transition is reversible on decompression.
\end{abstract}

\section{Introduction}

In recent years, there has been intense activity, both experimental and theoretical, in exploring fascinating physical properties of newer forms of carbon, namely fullerenes and single wall carbon nanotubes (SWNT) [1]. These properties include high structural stability and novel electronic transport. It is hoped that SWNT will have potential applications in nanometer-sized electronics and in development of high strength polymeric composite materials. Initially fullerenes $\mathrm{C}_{60}$ and $\mathrm{C}_{70}$ had occupied a center-stage, but in recent years most of the attention is focussed on carbon nanotubes. After the first observation [2] of multiwall nanotubes in 1991, a major breakthrough occurred in 1996 when bundles of aligned SWNT with narrow-size distribution could be produced in large quantities by laser pulse vaporization [3], followed by electric arc method [4]. The SWNT in a bundle are arranged in nearly a two dimensional triangular lattice, with intertube spacing of $\approx 3.4 \AA$, typical of van der Waals interaction between the tubes. Recent scanning tunneling spectroscopy experiments [5] have verified the theoretical predictions that the electronic properties of SWNT are given by one-dimensional density of states. These isolated tubes can be semiconducting or metallic, depending on their diameter and the chiral angle defined by the orientation of the hexagons with respect to the nanotube axis. The chiral vector which connects two crystallographically equivalent sites on a $2 \mathrm{D}$ graphene sheet is $n \mathbf{a}_{1}+m \mathbf{a}_{2}$, where $\mathbf{a}_{1}$ and $\mathbf{a}_{2}$ are the unit 
vectors in the $2 \mathrm{D}$ hexagonal honeycomb lattice. For $n \neq m \neq 0$, the tube has chiral symmetry and achiral tubes with $m=0$ and $n=m$ are called zigzag and arm-chair tubes, respectively. The diameter of the SWNT is related to the $(n, m)$ values by $d=a\left[3\left(n^{2}+m^{2}+n m\right)\right]^{1 / 2} / \pi$, where $a$ is the nearest C-C distance $(=1.42 \AA)$. Isolated tubes are metallic when $(n-m) / 3$ is an integer. However, intertube interaction between the tubes in a bundle does introduce additional features like a pseudogap of about $0.1 \mathrm{eV}$ at the Fermi level in a bundle of metallic $(10,10)$ tubes [6] and enhanced density of states near the Fermi level [7].

The pseudogap is reduced due to orientational and other types of disorder [7]. The elastic properties of SWNT bundles are highly anisotropic [8]. The tubes can be easily distorted perpendicular to their axis whereas along the tube axis, the bundle will be very rigid because such distortions are analogous to in-plane distortions of graphite.

Hexagonal graphite has lowest internal energy and hence is the equilibrium structure of carbon at ambient conditions. Pressure-induced phase transitions under static and dynamic loading between the many allotropes of carbon like cubic diamond, hexagonal diamond, graphite, fullerenes $\mathrm{C}_{60}$ and $\mathrm{C}_{70}$ and their polymeric and amorphous forms are of great scientific interest and have practical importance.

Raman spectroscopy, in particular resonant Raman, has played a key role in understanding the vibrational and electronic properties of fullerenes and SWNT, especially the one-dimensional nature [9] of the latter. It was shown that the position and the shape of the radial breathing mode $\left(\approx 170 \mathrm{~cm}^{-1}\right)$ of SWNT depends on the exciting laser energy which can be explained in terms of a diameter-selective Raman scattering mechanism. We have earlier used Raman spectroscopy to explore the pressure and temperature induced orientational phase transitions in solid $\mathrm{C}_{60}$ and solid $\mathrm{C}_{70}[10,11]$, pressure induced amorphisation of solid $\mathrm{C}_{70}$ [12] and pressure-temperature induced polymeric phases $[13,14]$ of $\mathrm{C}_{60}$ and $\mathrm{C}_{70}$. The main focus of this paper is to discuss our recent results on pressure effects on SWNT bundles probed using Raman spectroscopy upto a maximum pressure of $25.9 \mathrm{GPa}$ in a diamond anvil cell [15]. After the completion of our work, we came across the work of Venkateswaran et al. [16] on SWNT bundles carried out upto $\approx 5 \mathrm{GPa}$. We will make comparisons of our data with the numerical simulations and data reported by Venkateswaran et al. [16]. In order to put our high pressure results on SWNT bundles in proper perspective, we shall briefly summarise in Section 2 the pressure results on graphite, solid $\mathrm{C}_{60}$ and $\mathrm{C}_{70}$. Section 3 contains our recent high pressure Raman results on SWNT bundles.

\section{Pressure Behaviour of Graphite and Fullerenes}

\subsection{Pressure effects on graphite}

The behaviour of graphite under static pressures has been studied by X-ray diffraction, Raman scattering, optical reflection and absorption techniques and the results vary, possibly due to the nature of the samples used and different pressure transmitting media [17]. X-ray diffraction studies of Yagi et al. [18] showed that graphite transforms to hexagonal diamond at $18 \mathrm{GPa}$ which reverts to graphite on release of pressure. Using He/xenon as pressure transmitters, Raman studies by Goncharov et al. [19] showed that the $1580 \mathrm{~cm}^{-1}$ in-plane $\mathrm{E}_{2 \mathrm{~g}}$ mode broadened significantly upto $35 \mathrm{GPa}$, beyond which the mode did not shift or broaden. These effects were reversible on decompression. 
However, in a later study carried out using helium as pressure transmitter on single crystal graphite produced by hot pressing pyrolytic graphite [20], a transition to the amorphous phase at $23 \mathrm{GPa}$ was inferred based on substantial broadening of the $1580 \mathrm{~cm}^{-1}$ Raman mode and changes in the absorption spectra in the visible and near infrared range. Optical reflectivity experiments showed [21] that the high pressure phase of graphite at $31 \mathrm{GPa}$ (expected to be amorphous) is completely reversible on release of pressure.

\subsection{Pressure effects on fullerenes $-C_{60}$ and $C_{70}$}

High pressure studies on fullerenes have addressed three issues: (i) the pressure dependence of the orientational ordering transition temperature [22], (ii) the effect on the bandgap $E_{\mathrm{g}}$ related to the highest occupied molecular orbital (HOMO) - lowest unoccupied molecular orbital (LUMO) gap [11,23], and (iii) the nature of the high pressure phases. Photoluminescence [23] and optical reflectivity [24] measurements have yielded $\mathrm{d} E_{\mathrm{g}} / \mathrm{d} P=-0.14 \mathrm{eV} / \mathrm{GPa}$ for solid $\mathrm{C}_{60}$. Under static hydrostatic pressures at room temperature, Raman and optical reflectivity studies have revealed that $\mathrm{C}_{60}$ transforms completely to the amorphous carbon phase around $22 \mathrm{GPa}$ which is irreversible on release of pressure [21]. On the other hand, the high pressure phase $(P>20 \mathrm{GPa})$ in solid $\mathrm{C}_{70}$ shows Raman signatures of amorphous carbon which reverts to crystalline $\mathrm{C}_{70}$ on decompression [11,12]. As the $\mathrm{C}-\mathrm{C}$ distance between the neighbouring molecules decreases, the intermolecular interactions (which is van der Waals type at ambient pressure) acquire some covalent character accompanied by a tendency of conversion from $\mathrm{sp}^{2}$ to $\mathrm{sp}^{3}$ hybridization. The molecular nature of the crystal can be gradually lost and this can explain why the intramolecular modes in solid $\mathrm{C}_{70}$ are not seen above $12 \mathrm{GPa}$ $\left(d_{\mathrm{C}-\mathrm{C}} \approx 2.82 \AA\right)$. At higher pressures $(P \approx 20 \mathrm{GPa})$, when the fraction of $\mathrm{sp}^{3}$ bonds has increased sufficiently, the vibrational signatures mimic those of amorphous carbon. The question of reversibility or irreversibility of the amorphous phase on decompression should be related to the relative strengths of the repulsive intermolecular interaction vis-à-vis the intramolecular interaction. If the former is sufficiently higher, the molecules can be permanently distorted/destroyed and the amorphous phase will be irreversible. This seems to be the case in solid $\mathrm{C}_{60}$ where the contact intermolecular distance becomes much smaller $(\approx 1.89 \AA)$ than in $\mathrm{C}_{70}[11]$.

Non-hydrostatic pressures in the diamond-anvil cell have led to the conversion of solid $\mathrm{C}_{60}$ to diamond [25]. Under shockwave conditions, solid $\mathrm{C}_{60}$ yields graphitic material [26]. Under simultaneous application of high pressures (upto $8 \mathrm{GPa}$ ) and high temperatures $\left(<750^{\circ} \mathrm{C}\right) \mathrm{C}_{60}$ molecules have been shown to polymerise by bonding through $(2+2)$ cycloaddition reaction of each molecule with its neighbours [27]. This is similar to the pressure-induced cross-linking reaction of unsaturated organic molecules to denser, more saturated species [28]. In the $1 \mathrm{D}$ orthorhombic phase, each $\mathrm{C}_{60}$ molecule is bonded to two neighbours in $\langle 110\rangle$ direction. When these chains are bonded to their next-nearest neighbours in the (001) plane or (111) plane, polymerised 2D phases were formed with tetragonal symmetry with four nearest neighbours or rhombohedral symmetry with six nearest neighbours, respectively. Very recently, X-ray diffraction experiments on $\mathrm{C}_{60}$ samples quenched from $13 \mathrm{GPa}$ with some non-hydrostatic component and $547^{\circ} \mathrm{C}$ have revealed a $3 \mathrm{D}$ polymeric phase [29]. In compression, under similar conditions, polymerisation is not observed in the case of solid $\mathrm{C}_{70}$ as studied by Raman 
and infrared measurements although X-ray diffraction does reveal a structural transition from the initial h.c.p. structure to a rhombohedral structure which recovers to an f.c.c. structure on annealing [13]. However, new infrared modes in high-pressure hightemperature treated samples which are found to be different from that for photopolymerised $\mathrm{C}_{70}$ have been attributed to the formation of $\mathrm{C}_{70}$ dimers [14]. In order to understand the lack of polymerisation in $\mathrm{C}_{70}$, we note that for the polymerisation reaction to occur in $\mathrm{C}_{70}$, as is the case of $\mathrm{C}_{60}$, it is necessary that the parallel configuration of $\mathrm{C}=\mathrm{C}$ double bonds of the neighbouring molecules be brought close together under compression. In addition, it has been argued that there are topochemical constraints in the polymerisation of $\mathrm{C}_{70}$, in that only the double bonds on the polar caps of the molecules are reactive, whereas the cyclic double bonds on the equatorial belt are ineffective in undergoing $(2+2)$ cycloaddition reaction.

\section{Pressure Effects on SWNT Bundles}

We have carried out room-temperature Raman measurements on SWNT bundles upto 25.9 GPa using a diamond-anvil cell with 16:3:1 methanol-ethanol-water mixture as pressure transmitting medium. The spectra were recorded using SPEX spectrometer equipped with a cooled photomultiplier tube. SWNT bundles were prepared by the carbon arc method, similar to that of Journet et al. [4]. Fig. 1a shows Raman spectra attributed to the radial mode of SWNT excited using $5145 \AA$ laser radiation. Curve 1 is the Raman spectrum of the sample in air at ambient pressure. Theoretical calculations [30] using the force constant model show that the frequency of the radial mode depends sensitively on the tube diameter (independent of their types) given by an empirical relation $\omega_{\mathrm{R}}\left(\mathrm{cm}^{-1}\right)=223.75 / d(\mathrm{~nm})$. This relation gives $\omega_{\mathrm{R}}=164.8 \mathrm{~cm}^{-1}$ for the $(10,10)$ tube and $183.1 \mathrm{~cm}^{-1}$ for the $(9,9)$ tube. These numbers are different from the recently done numerical simulations using the generalised tight-binding molecular dynamics scheme (GTBMD) [16]. The inclusion of van der Waals interaction between the tubes shifts the radial mode frequency from $171.8 \mathrm{~cm}^{-1}$ to $186.2 \mathrm{~cm}^{-1}$ for the $(9,9)$ tubes, i.e. the radial mode frequency blue shifts by $14.4 \mathrm{~cm}^{-1}$ due to intertube interactions. Assuming that this blue shift is independent of the tube diameter [16], we suggest an empirical relation for the radial mode frequency of tubes in a rope as $\omega_{\mathrm{R}}\left(\mathrm{cm}^{-1}\right)=14.4+209.9 / d(\mathrm{~nm})$, which retains the $1 / d$ dependence of $\omega_{\mathrm{R}}$ and reproduces the GTBMD results for the $(9,9)$ tubes in a bundle. Taking a clue from the earlier observation that $(10,10)$ tubes are most abundantly produced [31], we believe that the radial mode at $170 \mathrm{~cm}^{-1}$ is associated with similar tubes in our sample. Curve 3 in Fig. 1a shows the Raman spectrum of the sample at ambient pressure but soaked in methanol-ethanol-water mixture. For comparison, the Raman spectrum of the sample in air has also been plotted as curve 2 (which is same as curve 1). Curve 4 is the Raman spectrum of the sample recovered after pressure cycling from $25.9 \mathrm{GPa}$. We clearly note that the mode frequencies are slightly higher in curve 3 as compared to the ones in curve 2. This can happen if the alcohol mixture intercalates between the tubes in the bundle because the presence of alcohol can offer some resistance to the radial motion of the carbon atoms of the SWNT. Fig. 1b shows Raman spectra in the tangential mode region at a few typical pressures, namely, $P=0,7.9,11.5$ and $22.7 \mathrm{GPa}$. The modes with peaks at 1554 and $1569 \mathrm{~cm}^{-1}$ were assigned [9] to modes of $E_{2 \mathrm{~g}}$ symmetry, $1594 \mathrm{~cm}^{-1}$ is associated with unresolved doublet $A_{1 \mathrm{~g}}+E_{1 \mathrm{~g}}$ and $1606 \mathrm{~cm}^{-1}$ with $\mathrm{E}_{2 \mathrm{~g}}$ symmetry. Our main results are as follows. 


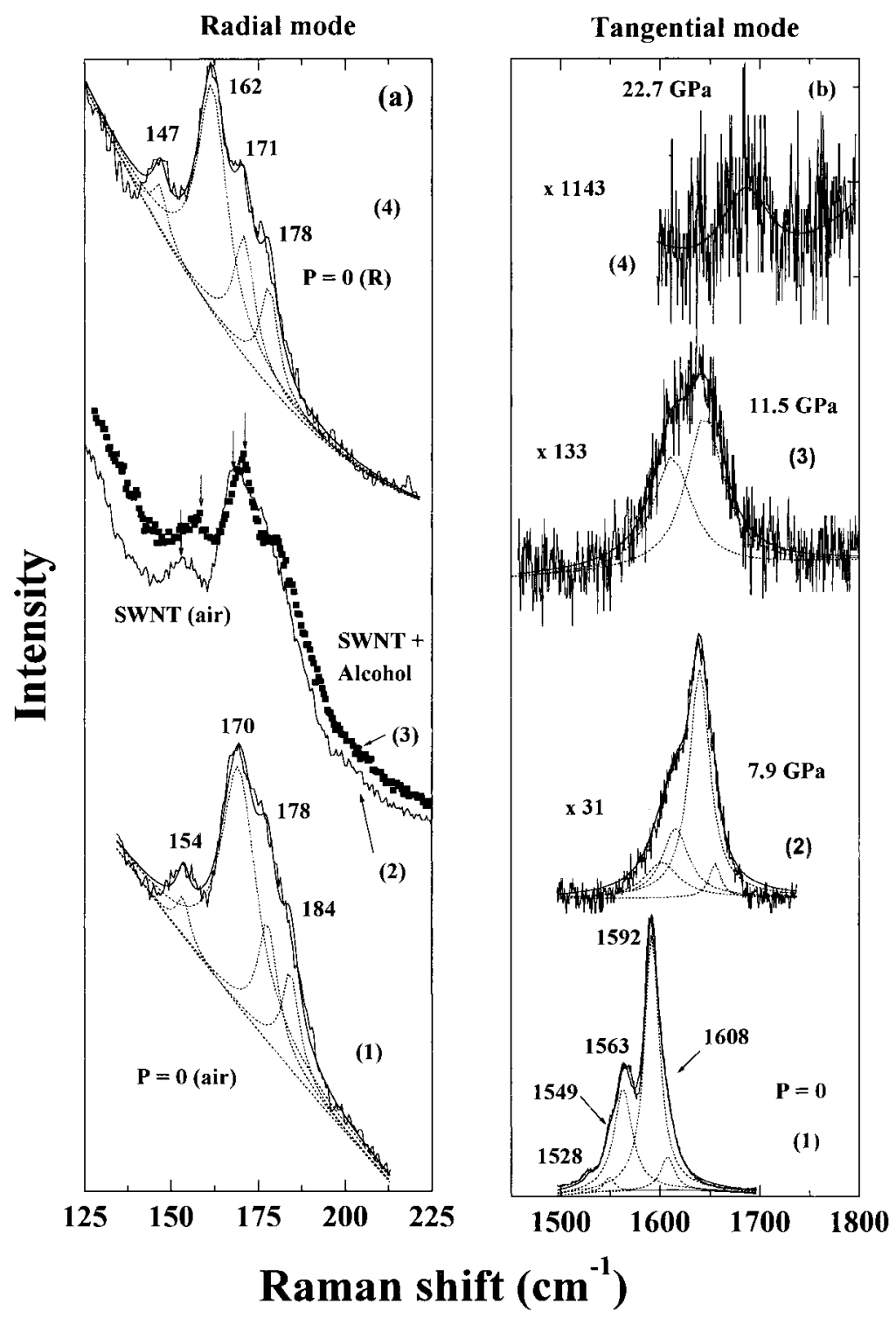

Fig. 1. a) Raman spectra in the radial mode region at ambient pressure. Curves 1 and 2: SWNT bundle in air; curve 3: sample soaked in alcohol mixture. The arrows are shown to highlight the blue shift of curve 3 with respect to curve 2. Curve 4: sample recovered after pressure cycle from 25.9 GPa. The peak positions of the fitted Lorentzians are marked. b) Tangential mode region in increasing pressure cycle. Curve 1: at $0 \mathrm{GPa}$; curve 2: at $7.9 \mathrm{GPa}$; curve 3 : at $11.5 \mathrm{GPa}$; curve 4 : at $22.7 \mathrm{GPa}$. The corresponding multiplication factors for the intensities compared to that of curve 1 are mentioned to the left of each of the curves

1. The intensities of the radial modes fall rapidly with pressure and the spectra could be recorded only till $2.6 \mathrm{GPa}$. Fig. 2 shows the pressure dependence of the radial mode frequency in the increasing pressure run and the results for the decreasing pressure 


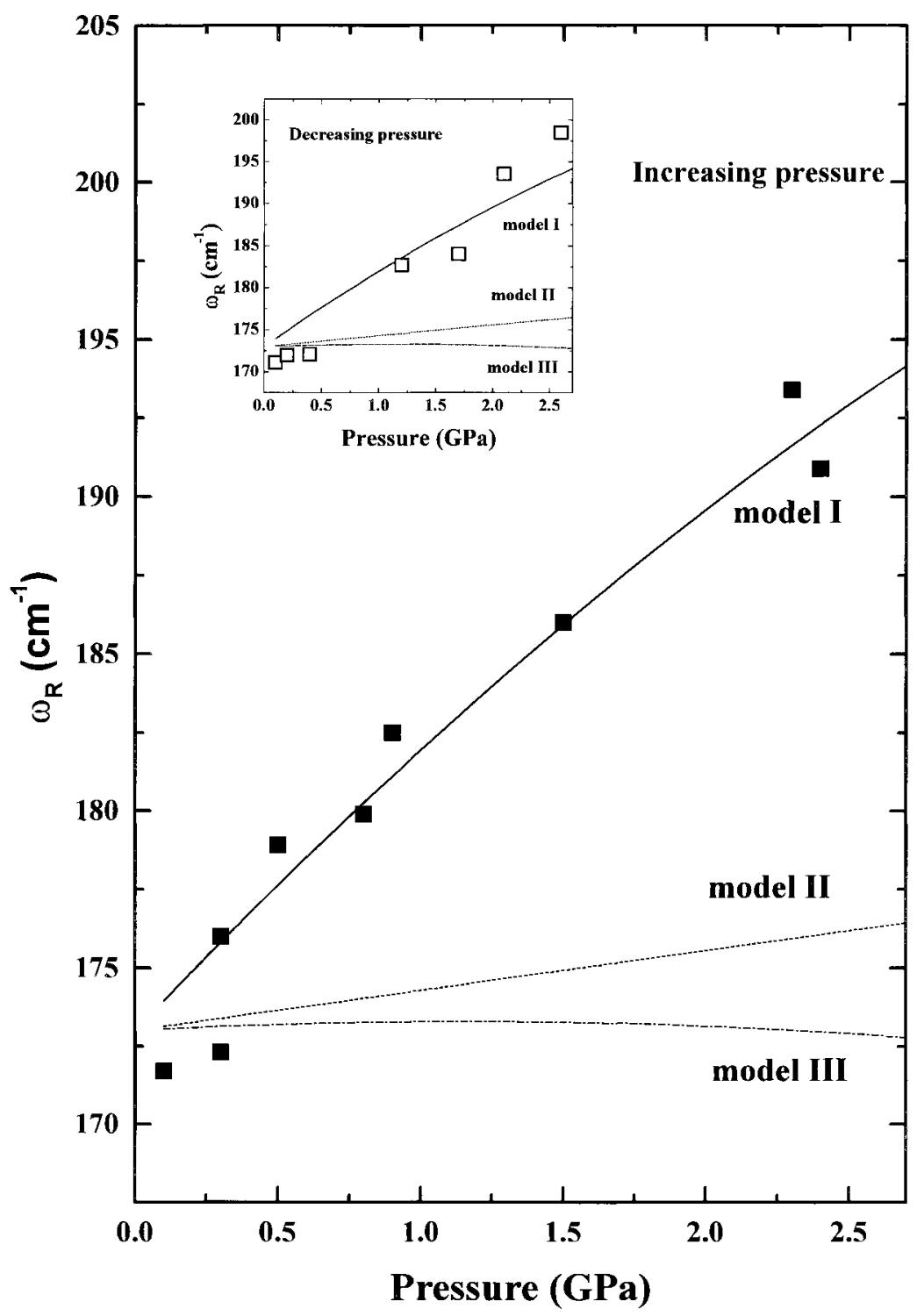

Fig. 2. Pressure dependence of the radial mode frequency in increasing pressure runs. The lines represent the calculated dependence in three different models (Ref. [16]). Inset shows the results for the decreasing pressure cycle

cycle are shown in the inset. The lines represent calculations of Ref. [16] based on three different models. In model I, the entire triangular tube lattice is uniformly compressed radially which corresponds to the situation that the pressure transmitting medium is not present into the interstitial channels in the tube lattice. This is not the case in models II and III where all the individual nanotubes in a triangular lattice feel the pressure medium. However, in model II, intertube interactions are ignored and therefore the com- 
pression is symmetric for all tubes whereas these interactions are included in model III. Like in Ref. [16], the pressure dependence of the radial mode is consistent only with model I. This is rather surprising because the non-penetration of the pressure transmitting fluid inside the bundle as supposed in model I is inconsistent with the shift of the mode frequency in Fig. 1a (cf. curves 2 and 3).

2. In the tangential mode regime as well, the intensities of the modes decrease and lines broaden significantly with pressure. Upto $10 \mathrm{GPa}$, the data can be resolved into four modes, beyond which it could be fitted with two Lorentzian functions upto $16 \mathrm{GPa}$. At higher pressures, the peaks are very weak and broad (cf. Fig. 1b). Interest-

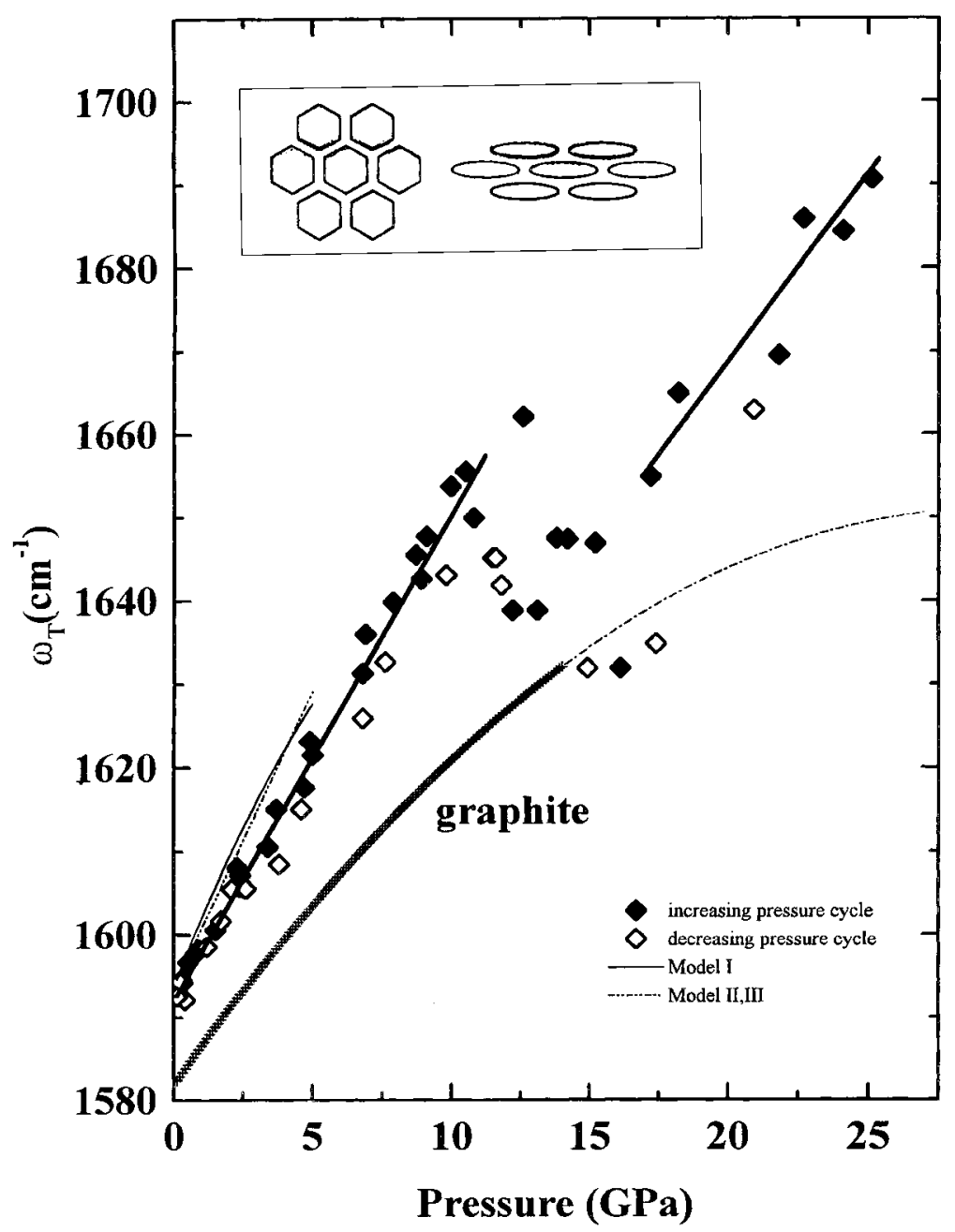

Fig. 3. Pressure dependence of the strongest tangential mode frequency. The thick solid lines are least square fitted, giving $\mathrm{d} \omega / \mathrm{d} P=5.3 \mathrm{~cm}^{-1} / \mathrm{GPa}(0$ to $10 \mathrm{GPa})$ and $4.5 \mathrm{~cm}^{-1} / \mathrm{GPa}$ (16 to $\left.26 \mathrm{GPa}\right)$. The calculated values for three different models (Ref. [16]) are also shown. The pressure dependence of the $1580 \mathrm{~cm}^{-1}$ mode of graphite (Ref. [33]) is also shown. The inset shows the faceting of SWNT in a bundle leading to hexagonal and elliptical cross-section 
ingly, on decompression, all the features - lineshapes, intensities, linewidths and peak positions - are more or less reversible. Fig. 3 shows the pressure variation of the most intense peak in increasing pressure (solid diamonds) as well as decreasing pressure (open diamonds) cycles. We have also plotted the predictions of model I, II and III of Ref. [16] in Fig. 3. The pressure dependence of the tangential modes in all the three models is similar till $5 \mathrm{GPa}$, in contrast to the behaviour of the radial mode in three different models. We note that the simulations [16] were done only till $5 \mathrm{GPa}$ and may not be fully valid at high pressures, where intertube interactions should include additional contributions due to electron hopping between the tubes, in addition to the usual Lennard-Jones form for the van der Waals interactions between the tubes [32]. Most interesting feature in Fig. 3 is the observed decrease in mode frequency between about 11 and $16 \mathrm{GPa}$. We do not understand the origin of this decrease.

Structural properties of a carbon nanotube crystal have been examined theoretically using the valence-force model to treat the atomic interactions within each tube and van der Waals interactions between the tubes in terms of the parametrised 6-12 potential. It is seen that for tube diameter, above $25 \AA$, the tubes flatten against each other forming a honeycomb structure [8]. In order to explain an exceptionally large and reversible volume reduction of SWNT ropes in high pressure experiments carried out upto $2.9 \mathrm{GPa}$, it has been suggested that under pressure, smaller diameter tubes can also facet and the tube cross-section can become hexagonal to elliptical [34] as depicted in the inset of Fig. 3. This can result in the considerable overlap of the surfaces of neighbouring tubes, mimicking the situation in graphite. We have plotted the experimentally determined [33] pressure dependence of the graphite $E_{2 g} 1580 \mathrm{~cm}^{-1}$ mode in Fig. 3. The experimental data in Ref. [33] obtained till $14 \mathrm{GPa}$ were fitted by $\omega(P)=\omega_{0}\left[\left(\delta_{0} / \alpha\right) P+1\right]^{\alpha}$, where $\omega_{0}=1579 \mathrm{~cm}^{-1}$, $\delta_{0}=2.96 \times 10^{-3} / \mathrm{GPa}$ and $\alpha=0.080$. We see that the frequency of the Raman mode in graphite lies below that of the main peak in SWNT bundle and the pressure derivatives are similar for graphite and our sample at higher pressures (>16 GPa). This leads us to conjecture that the cross-sections of the SWNT in bundles deform considerably at $\approx 11 \mathrm{GPa}$ resulting in overlap of surfaces of the tubes whose Raman signatures are like those of graphite like carbon. An irrevesible transition from SWNT bundles to graphite has been inferred in the unpublished reports of Kuznetsov and Fischer mentioned in Ref. [34]. However, our experiments show that the pressure effects are completely reversible on release of pressure. Such resilience of SWNT ropes under hydrostatic pressure is really remarkable and needs to be probed by X-ray diffraction as well as to be understood theoretically.

Acknowledgement A.K.S. and P.V.T. would like to thank the Department of Science and Technology and CSIR India for financial assistance.

\section{References}

[1] M.S. Dresselhaus, G. Dresselhaus, and P.C. EkLund, Science of Fullerenes and Carbon Nanotubes, Academic Press, London/New York 1996.

[2] S. IJIMA, Nature (London) 354, 56 (1991).

[3] A.Thess, R. Lee, P. Nikolaev, H. Dai, P. Petit, J. Robert, C. Xu, Y.H. Lee, S.G. Kim, A.G. Rinzler, D.T. Colbert, C.E. Scuseria, D. Tomanek, J.E. Fischer, and R.E. SMalley, Science 273, 483 (1996).

[4] C. Journet, W.K. Maser, P. Bernier, A. Loiseau, M. Lamy de la Chapelle, S. Lefrant, P. DeNIARD, R. LEE, and J.E. FISCHER, Nature (London) 388, 756 (1997). 
[5] J .W. C. Wildoer, L.C. Venema, A.G. Rinzler, R.E. Smalley, and C. DekKer, Nature (London) 391, 59 (1998).

T.W. Odom, J.L. Huang, P. Kim, and C.M. Lieber, Nature (London) 391, 62 (1998).

[6] P. Delaney, H.J. Choi, J. Ihm, S.G. Louie, and M.L. Cohen, Nature (London) 391, 466 (1998).

[7] Y.K. Kwon, D. TomaneK, Y. H. Lee, K. H. Lee, and S. Saito, J. Mater. Res. 13, 2363 (1998).

[8] J. Tersoff and R.S. RuOFF, Phys. Rev. Lett. 73, 676 (1994).

[9] A.M. Rao, E. Richter, S. Bandow, B. Chase, P.C. Eklund, K.A. Williams, S. Fang, K.R. Subbaswamy, M. Menon, A. Thess, R.E. Smalley, G. Dresselhaus, and M.S. Dresselhaus, Science 275, 187 (1997).

[10] N. Chandrabhas, M.N. Shashikala, D.V.S. Muthu, A.K. Sood, and C.N.R. Rao, Chem. Phys. Lett. 197, 319 (1992).

N. Chandrabhas, K. Jayram, D.V.S. Muthu, A.K. Sood, R. Seshadri and C.N.R. Rao, Phys. Rev. B 47, 10963 (1993).

S.K. Ramasesha, A.K. Singh, R. Seshadri, A.K. Sood, and C.N.R. Rao, Chem. Phys. Lett. 220, 203 (1994).

[11] A.K. Sood, N. Chandrabhas, D.V.S. Muthu, Y. Hariharan, A. Bharathi, and C.S. Sunder, Phil. Mag. B 70, 347 (1994).

[12] N. Chandrabhas, A.K. Sood, D.V.S. Muthu, C.S. Sunder, A. Bharathi, Y. Hariharan, and C.N.R. RaO, Phys. Rev. Lett. 73, 3411 (1994).

[13] C.S. Sunder, P.Ch. Sahu, V.S. Sastry, G.V.N. Rao, V. Sridharan, M. Premila, A. Bharathi, Y. Hariharan, T.S. Radhakrishnan, D.V.S. Muthu, and A.K. Sood, Phys. Rev. B 53, 8180 (1996).

[14] M. Premila, C.S. Sunder, P.Ch. Sahu, A. Bharathi, Y. Hariharan, D.V.S. Muthu, and A.K. Sood, Solid State Commun. 104, 237 (1997).

[15] Pallavi V. Teredesai, A.K. Sood, D.V.S. Muthu, R. Sen, A. Govindaraj, and C.N.R. Rao, unpublished.

[16] U.D. Venkateswaran, A.M. Rao, E. Richter, M. Menon, A. Rinzler, R.E. Smalley, and P.C. EKLUND, Phys. Rev. B 59, 10928 (1999).

[17] S.M. SHARMA and S.K. SiKKa, Progr. Mater. Sci. 40, 1 (1996).

[18] T. Yagi, W. Utsumi, M. Yamanaka, T. Kikegawa, and O. Shimomura, Phys. Rev. B 46, 6031 (1992).

[19] A.F. Goncharov, I.N. Makarenko and S.M. Stishov, Soviet Phys. - J. Exper. Theor. Phys. 69, 380 (1989).

[20] A.F. Goncharov, Soviet Phys. - J. Exper. Theor. Phys. Lett. 51, 418 (1990).

[21] D.W. Snoke, Y.S. Raptis, and K. Syassen, Phys. Rev. B 45, 14419 (1992).

[22] G.A. Samara, J.E. Schirber, B. Morosin, L.V. Hansen, D. Loy, and A.P. Sylwester, Phys. Rev. Lett. 67, 3136 (1991).

[23] A.K. Sood, N. Chandrabhas, D.V.S. Muthu, A. Jayraman, N. Kumar, H.R. Krishnamurthy, T. PradeEP, and C.N.R. RaO, Solid State Commun. 81, 89 (1992).

[24] D.W. Snoke, K. Syassen, and A. Mittelbach, Phys. Rev. B 47, 4146 (1993).

[25] M. Nunez-Regueiro, P. Monceau, and J.-L. Hodeau, Nature (London) 355, 237 (1992).

[26] C.S. Yoo and W.J. Nellis, Science 254, 1489 (1991).

[27] Y. IwASA et al., Science 264, 1570 (1994).

M. NunEz-Regueiro et al., Phys. Rev. Lett. 74, 278 (1995).

[28] M. Nicol, and G.Z. YIn, J. Phys. (France) 45, C8-163 (1984).

[29] L. Marques, M. Mezouar, J.-L. Hodeau, M. Nunez-Regueiro, N.R. Serebryanaya, V.A. IvdenKo, V.D. Blank, and G.A. Dubitsky, Science 283, 1720 (1999).

[30] S. Bandow, and S. Asaka, Phys. Rev. Lett. 80, 3779 (1998).

[31] J.M. Cowley, P. Nikolaev, A. Thess, and R. E. Smalley, Chem. Phys. Lett. 265, 379 (1997).

[32] E. RICHTER, private communications.

[33] M. Hanfland, H. Beister, and K. Syassen, Phys. Rev. B 39, 12598 (1989).

[34] S.A. Chesnokov, V.A. Nalimova, A.G. Rinzler, R.E. Smalley, and J.E. Fischer, Phys. Rev. Lett. 82, 343 (1999). 
